\title{
Outage Analysis of Train-to-Train Communication Model over Nakagami- $m$ Channel in High-Speed Railway
}

\author{
Pengyu Liu, ${ }^{1}$ Xiaojuan Zhou, ${ }^{2}$ and Zhangdui Zhong ${ }^{1}$ \\ ${ }^{1}$ State Key Laboratory of Rail Traffic Control and Safety, Beijing Jiaotong University, Beijing 100044, China \\ ${ }^{2}$ Department of Telecommunication Engineering, University of Armed Police Force Engineering, Xian 710086, China \\ Correspondence should be addressed to Pengyu Liu; 11111034@bjtu.edu.cn
}

Received 22 July 2012; Accepted 5 October 2012

Academic Editor: Ai Bo

Copyright (c) 2013 Pengyu Liu et al. This is an open access article distributed under the Creative Commons Attribution License, which permits unrestricted use, distribution, and reproduction in any medium, provided the original work is properly cited.

\begin{abstract}
This paper analyzes the end-to-end outage performance of high-speed-railway train-to-train communication model in highspeed railway over independent identical and nonidentical Nakagami- $m$ channels. The train-to-train communication is intertrain communication without an aid of infrastructure (for base station). Source train uses trains on other rail tracks as relays to transmit signals to destination train on the same track. The mechanism of such communication among trains can be divided into three cases based on occurrence of possible-occurrence relay trains. We first present a new closed form for the sum of squared independent Nakagami- $m$ variates and then derive an expression for the outage probability of the identical and non-identical Nakagami- $m$ channels in three cases. In particular, the problem is improved by the proposed formulation that statistic for sum of squared Nakagami- $m$ variates with identical $m$ tends to be infinite. Numerical analysis indicates that the derived analytic results are reasonable and the outage performance is better over Nakagami- $m$ channel in high-speed railway scenarios.
\end{abstract}

\section{Introduction}

Railway has played a significant role in helping transport passengers and goods. Accidents in railway always result in loss of lives and property. Safety issues have drawn increasing research attention due to the personal and property security.

A part of railway accidents is brought by malfunction of control center system. The train-to-train communication will serve as an assisting role which coordinates operations among trains based on multihop, when control center system is broken. It aims at detecting a potential collision and then broadcasting prewarning messages concerning this emergency to other trains on the same and neighboring tracks.

The multihop train-to-train communication model in physical layer lies in the thoughts that the source train uses the trains operating on other tracks as relays to transmit signals to destination train on the same track. Such mechanism can be divided into three cases based on occurrence of other relay trains. These relay trains occur following the Poisson Process [1], and therefore the arrival procedure follows the distribution of negative exponent. The proposed train-totrain communication model, introducing OFDM and MIMO technique, realizes the intertrain adhoc communication based on the Poisson Process in high-speed railway scenarios.

Since 2006, train-to-train communication has been researched by several organizations, such as the German Aerospace Center (DLR). Reference [2] discusses the RCAS approach consisting only of mobile adhoc components without the necessity of extensions of the railway infrastructure, while [3] describes an overview of the state of the art in collision avoidance related with transportation systems for maritime transportation, aircraft, and road transportation, and the RCAS is introduced. Reference [4] proposes a channel model for direct train-to-train communication appropriate for the $400 \mathrm{MHz}$ band, and [5] presents an infrastructure-less cross-layer train-to-train communication system exploiting all characteristics of a pervasive computing system, like direct communication in mobile adhoc networks. Reference [6] designs an infrastructure-less adhoc inter-vehicle communication system that fulfills these 
requirements with respect to the boundary conditions in the railway environment. Reference [7] presents analysis and results of a comprehensive measurement campaign investigating the propagation channel in case of direct communication between railway vehicles.

Despite that the fact the RCAS designed by the DLR has progressed tremendously in physical layer, it only work well for the train operation velocity lower than $200 \mathrm{Km} / \mathrm{h}$, which is not able to function in the high-speed railway. Generally speaking, the velocity of high-speed railway train is up to $360 \mathrm{Km} / \mathrm{h}$. In this case, safety distance among trains is 10 $\mathrm{Km}$ [8]. The proposed train-to-train communication model in physical layer has been evaluated by BER previously. In this paper, we continue to analyze the proposed multihop trainto-train communication model using outage probability. We first present a new closed-form for the sum of squared independent Nakagami- $m$ variates with identical $m$ [9], the proposed formulation improves the problem that statistic for sum of squared Nakagami- $m$ variables with identical $m$ is infinite. Then we derive an expression for the outage probability of the identical $m(m=1$ or $m=2)$ and nonidentical Nakagami- $m$ channels [10] in three cases. Such outage analysis is first applied to the multihop train-to-train communication model. The previous research indicates that the BER of train-to-train communication model reaches $10^{-6}$ when receiving SNR is $10 \mathrm{~dB}$, which meets the requirements of the International Union of Railway (UIC). Therefore the threshold should be set to $10 \mathrm{~dB}$. If the receiving signal SNR is below that value, the signal quality at receiver cannot realize normal communication among trains and the trainto-train communication is regarded as outage. The maximum distance of train-to-train communication is set to $6 \mathrm{Km}$ and it is probable that $m$ value of Nakagami- $m$ channel in two receiving path at receiver is different, for example, one path is $m=1$ and the other path is $m=2$. This paper considers the Nakagami- $m$ channel not only with identical $m$ but also with nonidentical $m$ in receiver's receiving path.

The rest of this paper is organized as follows. Section 2 gives a description of the proposed train-to-train communication model based on multihop. Section 3 derives expressions for the outage probability of three conditions. Section 4 shows the outage probability simulation results of this model. In Section 5, this paper is concluded.

\section{Proposed Train-to-Train Communication Model}

In this section, three cases of train-to-train communication model are presented in Figures 1, 2, and 3, where the S, $\mathrm{R} 1$, and $\mathrm{D}$ represent source terminal, fixed-occurring relay terminal, and destination terminal, respectively. The $\mathrm{R} 1$ is the train that meets $\mathrm{S}$ on another rail track, and the R2 and $\mathrm{R} 3$ represent the first possible-occurring relay terminal and the third possible-occurring relay terminal. The average operating velocities of the R1 and R2 are $100 \mathrm{~m} / \mathrm{s}$, and those of $\mathrm{D}$ and $\mathrm{R} 3$ are $-100 \mathrm{~m} / \mathrm{s}$. The rail 1 , rail 2 , rail 3 , and rail 4 stand for four parallel rail tracks. These cases are described below.
2.1. Case I. The $\mathrm{S}$ transmits signals to the $\mathrm{R} 1$ on different rail tracks when they meet each other. At the same time, the $S$ searches the potential relay R2 on a neighboring rail track 1 . If the R2 is not found, R1 will keep broadcasting messages within its communication coverage $(6 \mathrm{Km})$, and if it receives the response of $\mathrm{D}$, their communication link will be held on and the transmission between them is performed.

2.2. Case II. If the $\mathrm{R} 2$ is searched, the $\mathrm{S}$ and $\mathrm{R} 1$ simultaneously transmit the signals to the R2. The R2 also performs the search of the potential relay $\mathrm{R} 3$. If the $\mathrm{R} 3$ does not exist, it will operate for some seconds until the distance between R2 and D is within a communication range. Finally, the R2 and R1, acting as two relays of the source, will transmit the signals to the destination terminal.

2.3. Case III. If the $\mathrm{R} 3$ exists, it will receive the signals from the R2 and R1 and then combine them. Finally, the R3, R2, and R1, as three relays of the source, will forward the signals to the destination terminal.

Under these cases, this paper respectively analyzes the outage probability of the train-to-train communication model.

\section{Outage Analysis of Train-to-Train Communication Model}

$\left\{X_{n}, n=1, \ldots, M\right\}$ is $M$ independent Nakagami- $m$ distributed RVs, with PDF expressed as [11]

$$
f_{X l}(x)=\frac{2 x^{2 m_{n}-1} m_{n}^{m_{n}}}{\bar{x}_{n}^{m_{n}}(m-1) !} \exp \left(-\frac{m_{n} x^{2}}{\bar{x}_{n}}\right),
$$

where $m_{n}$ denotes the Nakagami- $m$ fading parameter and $\bar{x}_{n}$ is expectation of $X_{n}^{2}$.

Furthermore, let $Y_{n}=X_{n}^{2}$, the PDF given by

$$
f_{Y l}(x)=\frac{x^{m_{n}-1} m_{n}^{m_{n}}}{\bar{x}_{n}^{m_{n}}(m-1) !} \exp \left(-\frac{m_{n} x}{\bar{x}_{n}}\right)
$$

and $\mathrm{CDF}[9]$

$$
F_{Y l}(x)=1-\exp \left(-\frac{m_{n} x}{\bar{x}_{n}}\right) \sum_{l}^{m_{n}-1}\left(\frac{x m_{n}}{\bar{x}_{n}}\right)^{l} .
$$

Before analyzing the outage probability of train-to-train communication model, we first give the close-form of the sum of squared Nakagami- $m$ variates with identical $m$ and nonidentical $m$.

Theorem 1 (PDF of the Sum of Squared Nakgami- $m$ RVs with identical $m)$. Let $\left\{Y_{n}, n=1, \ldots, M\right\}$ be a set of $R V$ s following the PDF presented in (2), with $\bar{x}_{i}-\bar{x}_{j}=o(x), i \neq j$ and $m_{1}=$ $m_{2}=\cdots=m_{M}=m$. The PDF of the sum

$$
Z_{M}=\sum_{i=1}^{M} Y_{i}
$$


is given by

$$
\begin{aligned}
f_{Z_{M}}(z)= & \left(\frac{m}{\bar{z}}\right)^{(M-1) m} \frac{\Gamma(m)}{\Gamma(M m)} z^{(M-1) m} \\
& \times \frac{z^{m-1} m^{m}}{\bar{z}_{M}^{m}(m-1) !} \exp \left(-\frac{m z}{\bar{z}_{M}}\right) .
\end{aligned}
$$

Proof. Step 1. For $M=2$, the PDF of $Z_{2}=Y_{1}+Y_{2}$ can be evaluated as

$$
f_{Z_{2}}(z)=\int_{0}^{z} f_{Y_{1}}\left(x ; m_{1}, \bar{x}_{1}\right) f_{Y_{2}}\left(z-x ; m_{2}, \bar{x}_{2}\right) d x
$$

Insert (2) into (6) and use [12, equation (3.383.1)], (6) can be derived as

$$
\begin{aligned}
f_{Z_{2}}(z)= & \frac{B\left(m_{1}, m_{2}\right)}{\bar{z}_{1}^{m_{1}} \bar{z}_{2}^{m_{2}} \Gamma\left(m_{1}\right) \Gamma\left(m_{2}\right)} z^{-1+m_{1}+m_{2}} \exp \left(-\frac{m_{2} z}{\bar{z}_{2}}\right) \\
& * F_{1}\left(m_{1} ; m_{1}+m_{2} ;-\left(\frac{m_{1}}{\bar{z}_{1}}-\frac{m_{2}}{\bar{z}_{2}}\right) z\right)
\end{aligned}
$$

Since $m_{1} / z_{1}-m_{2} / \bar{z}_{2}=o(x)$ and using [12, equation (9.210.1)]

$$
\begin{aligned}
F_{1}\left(m_{1} ; m_{1}+m_{2} ;-\left(\frac{m_{1}}{\bar{x}_{1}}-\frac{m_{2}}{\bar{x}_{2}}\right) z\right) \\
=\sum_{k=0}^{+\infty} \frac{z^{k}}{k !} \frac{\left(m_{1}+k-1\right) ! /\left(m_{1}-1\right) !}{\left(m_{2}+m_{1}+k-1\right) ! /\left(m_{1}+m_{2}-1\right) !} \\
\quad \times\left[-\left(\frac{m_{1}}{\bar{z}_{1}}-\frac{m_{2}}{\bar{z}_{2}}\right) z\right]^{k}
\end{aligned}
$$

where $\left.\left[-\left(m_{1} / z_{1}-m_{2} / \bar{z}_{2}\right) z\right]^{k}\right|_{k=0}=1$ and $\left[-\left(m_{1} / \bar{z}_{1}-m_{2} / \bar{z}_{2}\right)\right.$ $z]\left.^{k}\right|_{k \neq 0}=0$, (7) can be denoted as

$$
\begin{aligned}
f_{Z_{2}}(z)= & \frac{B\left(m_{1}, m_{2}\right)}{\bar{x}_{1}^{m_{1}} \bar{x}_{2}^{m_{2}} \Gamma\left(m_{1}\right) \Gamma\left(m_{2}\right)} z^{-1+m_{1}+m_{2}} \exp \left(-\frac{m_{2} z}{\bar{z}_{2}}\right) \\
= & \left(\frac{m_{1}}{\bar{z}}\right)^{m_{1}}\left(\frac{m_{2}}{\bar{z}}\right)^{m_{2}} \frac{\Gamma\left(m_{2}\right)}{\Gamma\left(m_{2}+m_{1}\right)} \\
& \times z^{m_{1}} \frac{z^{m_{2}-1} m_{2}^{m_{2}}}{\bar{z}_{n}^{m_{2}}\left(m_{2}-1\right) !} \exp \left(-\frac{m_{2} z}{\bar{z}_{2}}\right) \\
= & \left(\frac{m_{1}}{\bar{z}}\right)^{m_{1}}\left(\frac{m_{2}}{\bar{z}}\right)^{m_{2}} \frac{\Gamma\left(m_{2}\right)}{\Gamma\left(m_{2}+m_{1}\right)} z^{m_{1}} f_{Y_{2}}(z) .
\end{aligned}
$$

Step 2. For $M=3$, the PDF of $Z_{3}$ can be efficiently expressed as

$$
f_{Z_{3}}(z)=\int_{0}^{z} f_{Z_{2}}\left(x ; m_{1}, \bar{x}_{1}\right) f_{Y_{3}}\left(z-x ; m_{3}, \bar{x}_{3}\right) d x .
$$

Following Step 1 of PDF calculation of $Z_{2}$, the PDF of $Z_{3}$ can be denoted as:

$$
\begin{aligned}
f_{Z_{2}}(z)= & \left(\frac{m_{1}}{\bar{z}}\right)^{m_{1}}\left(\frac{m_{2}}{\bar{z}}\right)^{m_{2}}\left(\frac{m_{3}}{\bar{z}}\right)^{m_{3}} \\
& \times \frac{\Gamma\left(m_{3}\right)}{\Gamma\left(m_{2}+m_{1}+m_{3}\right)} z^{m_{1}+m_{2}} f_{Y_{3}}(z) .
\end{aligned}
$$

Step 3. According to the same procedure as Steps 1 and 3, the sum of $M$ Nakagami- $m$ RVs can be expressed as

$$
f_{Z_{M}}(z)=\left(\frac{m z}{\bar{Y}_{1}}\right)^{m(M-1)} \frac{\Gamma(m)}{\Gamma(M m)} f_{Y_{M}}(z) .
$$

Corollary 2 (PDF of the sum of Squared Nakagami- $m$ RVs with identical $m$ ). The CDF of $Z_{M}$ is given by

$$
\begin{aligned}
F_{M}(Z)= & \left.\frac{m z}{\bar{Y}_{1}}\right)^{m(M-1)} \frac{\Gamma(m)}{\Gamma(M m)} \\
\times & {\left[\frac{((M-1) m-1) !}{\left(m / \bar{z}_{M}\right)^{M m}}-e^{-\left(m / \bar{z}_{M}\right) z}\right.} \\
& \left.\times \sum_{k=0}^{M m-1} \frac{((M-1) m-1) !}{k !} \frac{z^{k}}{\left(m / \bar{z}_{M}\right)^{M m-k}}\right] .
\end{aligned}
$$

Theorem 3 (PDF of the Sum of Squared Nakgami- $m$ RVs with nonidentical $m)$. Let $\left\{Y_{n}, n=1, \ldots, M\right\}$ be a set of $R V$ s following the PDF presented in (2), with $m_{1} \neq m_{2} \neq \cdots \neq m_{M} \neq m$. The PDF of the sum is given by [11]

$$
\begin{aligned}
f_{Z_{M}}(z)= & \sum_{i=1}^{M} \sum_{k=1}^{m_{i}} \frac{\bar{y}_{i}^{m_{i}} m_{h}^{m_{h}}}{\prod_{h=1}^{M} \bar{y}_{h}^{m_{h}} m_{i}^{m_{i}}} \\
& \times \prod_{j=1, j \neq i}^{M}\left(\frac{m_{j}}{\bar{y}_{j}}-\frac{m_{i}}{\bar{y}_{i}}\right)^{-m_{j}} \frac{z^{m_{i}-1} m_{i}^{m_{i}}}{\bar{y}_{i}^{m_{i}}(m-1) !} \exp \left(-\frac{m_{i} z}{\bar{y}_{i}}\right) \\
= & \sum_{i=1}^{M} \sum_{k=1}^{m_{i}} \frac{\bar{z}_{i}^{m_{i}}}{\prod_{h=1}^{M} \bar{y}_{h}^{m_{h}}} \prod_{j=1, j \neq i}^{M}\left(\frac{1}{\bar{y}_{j}}-\frac{1}{\bar{y}_{i}}\right)^{-m_{j}} F_{Y l}\left(x_{i}\right) .
\end{aligned}
$$

Corollary 4 (PDF of the sum of Squared Nakagami- $m$ RVs with nonidentical $m$ ). The CDF of $Z_{M}$ is given by [13]

$$
\begin{aligned}
F_{M}(Z)= & \sum_{i=1}^{M} \sum_{k=1}^{m_{i}} \frac{\bar{y}_{i}^{m_{i}} m_{h}^{m_{h}}}{\prod_{h=1}^{M} \bar{y}_{h}^{m_{h}} m_{i}^{m_{i}}} \prod_{j=1, j \neq i}^{M}\left(\frac{m_{j}}{\bar{y}_{j}}-\frac{m_{i}}{\bar{y}_{i}}\right)^{-m_{j}} \\
& \times\left(1-\exp \left(-\frac{m_{n} z}{\bar{y}_{n}}\right) \sum_{l}^{m_{n}-1}\left(\frac{z m_{n}}{\bar{y}_{n}}\right)^{l}\right) \\
= & \sum_{i=1}^{M} \sum_{k=1}^{m_{i}} \frac{\bar{y}_{i}^{m_{i}}}{\prod_{h=1}^{M} \bar{y}_{h}^{m_{h}}} \prod_{j=1, j \neq i}^{M}\left(\frac{1}{\bar{y}_{j}}-\frac{1}{\bar{y}_{i}}\right)^{-m_{j}} F_{Y l}(x i) .
\end{aligned}
$$






FIgURE 1: Train-to-train communication model of case I.

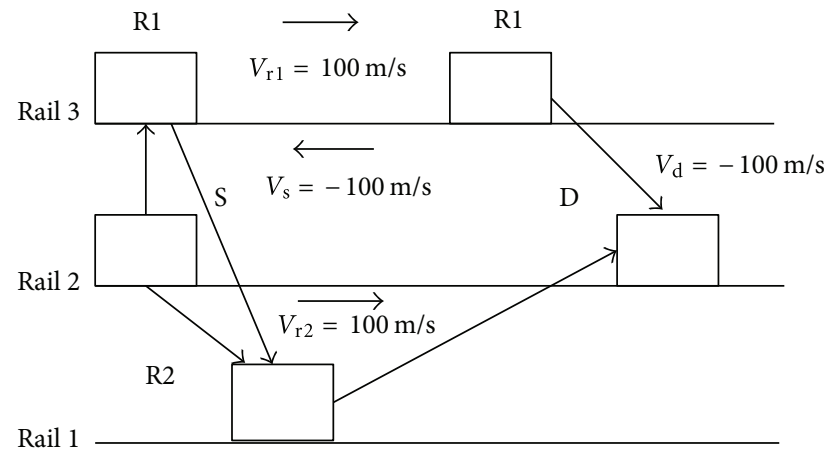

Figure 2: Train-to-train communication model of case II.

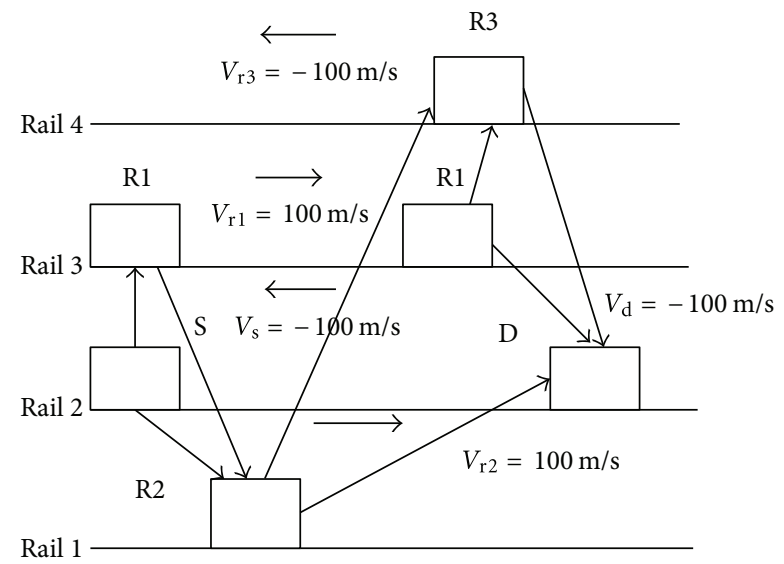

FIgURE 3: Communication model of case III.

The outage event happens when the $\mathrm{Y}$ falls below given threshold $\mathrm{Y}_{0}$ under Nakagami- $m$ channel and its probability is defined as

$$
\begin{aligned}
P_{\text {out }}(Y) & =\operatorname{Prob}\left[Y<Y_{0}\right] \\
& =1-\exp \left(-\frac{m_{n} Y_{0}}{\bar{y}}\right) \sum_{l}^{m_{n}-1}\left(\frac{Y_{0} m_{n}}{\bar{y}}\right)^{l},
\end{aligned}
$$

where $\bar{Y}$ is average receiving signal-to-noise ratio.

Next the outage probabilities of train-to-train communication model under three cases are analyzed.

3.1. Case I. In Figure $4, Y_{1}$ and $Y_{2}$ are SNR of receiving signal at $\mathrm{R} 1$ and $\mathrm{D}$, respectively. Since each node applies $2 * 2 \mathrm{MIMO}$,



FIGURE 4: Communication model of case I.

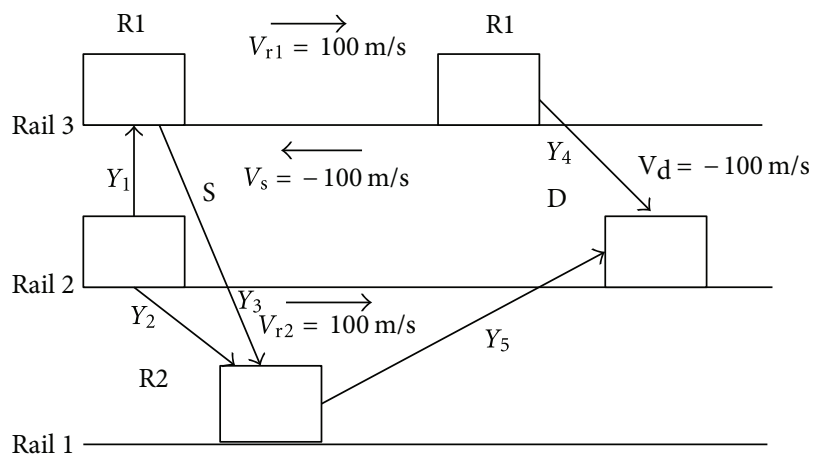

Figure 5: Communication model of case II.



FIgURE 6: Communication model of case III.

$Y_{1}$ and $Y_{2}$ are sum of 4 squared Nakagami- $m$ variables at receiver.

The outage probability $F_{\text {out }}$ of case I is expressed as

$F_{\text {outI }}=\operatorname{Prob}\left[Y_{1}<Y_{0}\right]+\operatorname{Prob}\left[Y_{1}>Y_{0}\right] * \operatorname{Prob}\left[Y_{2}<Y_{0}\right]$

$Y_{0}$ is the threshold SNR.

Inserting (13) or (15) into (17), the $F_{\text {outI }}$ under Nakagami- $m$ channel with identical $m$ and nonidentical $m$ can be written as follows. 
(1) Identical-m:

$$
\begin{aligned}
F_{\text {out I }}= & \left(\frac{m z}{\bar{Y}_{1}}\right)^{3 m} \frac{\Gamma(m)}{\Gamma(3 m)} \\
& \times\left[\frac{(3 m-1) !}{\left(m / \bar{Y}_{1}\right)^{3 m}}-\exp \left(-\left(\frac{m}{\bar{Y}_{1}}\right) Y_{0}^{k}\right)\right. \\
& \left.\times \sum_{k=0}^{3 m-1} \frac{(3 m-1) !}{k !} \frac{Y_{0}^{k}}{\left(m / \bar{Y}_{1}\right)^{4 m-k}}\right] \\
& \times\left[\frac{(3 m-1) !}{\bar{Y}_{2}}\right)^{3 m} \frac{\Gamma(m)}{\Gamma(3 m)}-\exp \left(-\left(\frac{m}{\bar{Y}_{2}}\right) Y_{0}^{k}\right) \\
& \left.\times \sum_{k=0}^{3 m-1} \frac{(3 m-1) !}{k !} \frac{Y_{0}^{k}}{\left(m / \bar{Y}_{2}\right)^{4 m-k}}\right] \\
& +\left[1-\left(\frac{m z}{\bar{Y}_{1}}\right)^{3 m} \frac{\Gamma(m)}{\Gamma(3 m)}\right. \\
& \times\left[\frac{(3 m-1) !}{\left(m / \bar{Y}_{1}\right)^{3 m}-\exp \left(-\left(\frac{m}{\bar{Y}_{1}}\right) Y_{0}^{k}\right.}\right) \\
& \left.\left.\times \sum_{k=0}^{3 m-1} \frac{(3 m-1) !}{k !} \frac{Y_{0}^{k}}{\left(m / \bar{Y}_{1}\right)^{4 m-k}}\right]\right]
\end{aligned}
$$

(2) Nonidentical $m$ :

$$
\begin{aligned}
F_{\text {out I }}= & \sum_{i=1}^{4} \sum_{k=1}^{m_{i}} \frac{\bar{Y}_{1}^{m_{i}}}{\prod_{h=1}^{M} \bar{Y}_{1}^{m_{h}}} \prod_{j=1, j \neq i}^{4}\left(\frac{1}{\bar{Y}_{j}}-\frac{1}{\bar{Y}_{i}}\right)^{-m_{j}} F_{Y l}\left(Y_{0}\right) \\
& +\left[1-\sum_{i=1}^{4} \sum_{k=1}^{m_{i}} \frac{\bar{Y}_{1}^{m_{i}}}{\prod_{h=1}^{4} \bar{Y}_{1}^{m_{h}}}\right. \\
& \left.\times \prod_{j=1, j \neq i}^{4}\left(\frac{1}{\overline{\bar{Y}}_{j}}-\frac{1}{\bar{Y}_{i}}\right)^{-m_{j}} F_{Y l}\left(Y_{0}\right)\right] \\
& * \sum_{i=1}^{4} \sum_{k=1}^{m_{i}} \frac{\bar{Y}_{2}^{m_{i}}}{\prod_{h=1}^{4} \bar{Y}_{2}^{m_{h}}} \prod_{j=1, j \neq i}^{4}\left(\frac{1}{\overline{\bar{Y}}_{j}}-\frac{1}{\bar{Y}_{i}}\right)^{-m_{j}} F_{Y l}\left(Y_{0}\right) .
\end{aligned}
$$

3.2. Case II. In Figure 5, $Y_{1}$ are SNR of receiving signal at $\mathrm{R} 1, Y_{2}, Y_{3}$ are SNR of receiving signal at $\mathrm{R} 2$, and $Y_{4}, Y_{5}$ are receiving signal SNR at $\mathrm{D}$.
The outage probability $F_{\text {outI }}$ of case I is expressed as

$$
\begin{aligned}
F_{\text {out II }}= & \operatorname{Prob}\left[Y_{1}<Y_{0}\right]+\operatorname{Prob}\left[Y_{1}>Y_{0}\right] \\
& * \operatorname{Prob}\left[Y_{2}+Y_{3}<Y_{0}\right]+\operatorname{Prob}\left[Y_{1}>Y_{0}\right] \\
& * \operatorname{Prob}\left[Y_{2}+Y_{3}>Y_{0}\right] * \operatorname{Prob}\left[Y_{4}+Y_{5}<Y_{0}\right] .
\end{aligned}
$$

Inserting (13) or (15) into (20), the $F_{\text {outII }}$ under Nakagami- $m$ channel with identical $m$ and nonidentical $m$ can be written as follows.

(1) Identical-m:

$$
\begin{aligned}
& F_{\text {out II }}=\left(\frac{m z}{\bar{Y}_{1}}\right)^{3 m} \frac{\Gamma(m)}{\Gamma(3 m)} \\
& \times\left[\frac{(3 m-1) !}{\left(m / \bar{Y}_{1}\right)^{3 m}}-\exp \left(-\left(\frac{m}{\bar{Y}_{1}}\right) Y_{0}^{k}\right)\right. \\
& \left.\times \sum_{k=0}^{3 m-1} \frac{(3 m-1) !}{k !} \frac{Y_{0}^{k}}{\left(m / \bar{Y}_{1}\right)^{4 m-k}}\right] \\
& +\left(\frac{m z}{\bar{Y}_{2}}\right)^{7 m} \frac{\Gamma(m)}{\Gamma(7 m)} \\
& \times\left[\frac{(7 m-1) !}{\left(m / \bar{Y}_{2}\right)^{7 m}}-\exp \left(-\left(\frac{m}{\bar{Y}_{2}}\right) Y_{0}^{k}\right)\right. \\
& \left.\times \sum_{k=0}^{7 m-1} \frac{(7 m-1) !}{k !} \frac{Y_{0}^{k}}{\left(m / \bar{Y}_{2}\right)^{8 m-k}}\right] \\
& \text { * }\left[1-\left(\frac{m z}{\bar{Y}_{1}}\right)^{3 m} \frac{\Gamma(m)}{\Gamma(3 m)}\right. \\
& \times\left[\frac{(3 m-1) !}{\left(m / \bar{Y}_{1}\right)^{3 m}}-\exp \left(-\left(\frac{m}{\bar{Y}_{1}}\right) Y_{0}^{k}\right)\right. \\
& \left.\left.\times \sum_{k=0}^{3 m-1} \frac{(3 m-1) !}{k !} \frac{Y_{0}^{k}}{\left(m / \bar{Y}_{1}\right)^{4 m-k}}\right]\right] \\
& +\left[1-\left(\frac{m z}{\bar{Y}_{1}}\right)^{3 m} \frac{\Gamma(m)}{\Gamma(3 m)}\right. \\
& \times\left[\frac{(3 m-1) !}{\left(m / \bar{Y}_{1}\right)^{3 m}}-\exp \left(-\left(\frac{m}{\bar{Y}_{1}}\right) Y_{0}^{k}\right)\right.
\end{aligned}
$$




$$
\begin{aligned}
& \left.\left.\times \sum_{k=0}^{3 m-1} \frac{(3 m-1) !}{k !} \frac{Y_{0}^{k}}{\left(m / \bar{Y}_{1}\right)^{4 m-k}}\right]\right] \\
& *\left[1-\left(\frac{m z}{\bar{Y}_{2}}\right)^{7 m} \frac{\Gamma(m)}{\Gamma(3 m)}\right. \\
& \times\left[\frac{(7 m-1) !}{\left(m / \bar{Y}_{2}\right)^{7 m}}-\exp \left(-\left(\frac{m}{\bar{Y}_{2}}\right) Y_{0}^{k}\right)\right. \\
& \left.\left.\times \sum_{k=0}^{7 m-1} \frac{(7 m-1) !}{k !} \frac{Y_{0}^{k}}{\left(m / \bar{Y}_{2}\right)^{8 m-k}}\right]\right] \\
& \text { * }\left[\left(\frac{m z}{\bar{Y}_{4}}\right)^{7 m} \frac{\Gamma(m)}{\Gamma(7 m)}\right. \\
& \times\left[\frac{(7 m-1) !}{\left(m / \bar{Y}_{4}\right)^{7 m}}-\exp \left(-\left(\frac{m}{\bar{Y}_{4}}\right) Y_{0}^{k}\right)\right. \\
& \left.\left.\times \sum_{k=0}^{7 m-1} \frac{(7 m-1) !}{k !} \frac{Y_{0}^{k}}{\left(m / \bar{Y}_{4}\right)^{8 m-k}}\right]\right] .
\end{aligned}
$$

(2) Nonidentical $m$ :

$$
\begin{aligned}
& F_{\text {out II }}=\sum_{i=1}^{4} \sum_{k=1}^{m_{i}} \frac{\bar{Y}_{1}^{m_{i}}}{\prod_{h=1}^{8} \bar{Y}_{1}^{m_{h}}} \prod_{j=1, j \neq i}^{4}\left(\frac{1}{\bar{Y}_{j}}-\frac{1}{\bar{Y}_{i}}\right)^{-m_{j}} F_{Y l}\left(Y_{0}\right) \\
& +\left[1-\sum_{i=1}^{4} \sum_{k=1}^{m_{i}} \frac{\bar{Y}_{1}^{m_{i}}}{\prod_{h=1}^{8} \bar{Y}_{1}^{m_{h}}}\right. \\
& \left.\times \prod_{j=1, j \neq i}^{4}\left(\frac{1}{\bar{Y}_{j}}-\frac{1}{\bar{Y}_{i}}\right)^{-m_{j}} F_{Y l}\left(Y_{0}\right)\right] \\
& * \sum_{i=1}^{8} \sum_{k=1}^{m_{i}} \frac{\bar{Y}_{2}^{m_{i}}}{\prod_{h=1}^{8} \bar{Y}_{2}^{m_{h}}} \prod_{j=1, j \neq i}^{8}\left(\frac{1}{\bar{Y}_{j}}-\frac{1}{\bar{Y}_{i}}\right)^{-m_{j}} F_{Y l}\left(Y_{0}\right) \\
& +\left[1-\sum_{i=1}^{4} \sum_{k=1}^{m_{i}} \frac{\bar{Y}_{1}^{m_{i}}}{\prod_{h=1}^{8} \bar{Y}_{1}^{m_{h}}}\right. \\
& \left.\times \prod_{j=1, j \neq i}^{4}\left(\frac{1}{\bar{Y}_{j}}-\frac{1}{\bar{Y}_{i}}\right)^{-m_{j}} F_{Y l}\left(Y_{0}\right)\right] \\
& *\left[1-\sum_{i=1}^{8} \sum_{k=1}^{m_{i}} \frac{\bar{Y}_{2}^{m_{i}}}{\prod_{h=1}^{8} \bar{Y}_{2}^{m_{h}}}\right.
\end{aligned}
$$

$$
\begin{gathered}
\left.\times \prod_{j=1, j \neq i}^{8}\left(\frac{1}{\bar{Y}_{j}}-\frac{1}{\bar{Y}_{i}}\right)^{-m_{j}} F_{Y l}\left(Y_{0}\right)\right] \\
* \sum_{i=1}^{8} \sum_{k=1}^{m_{i}} \frac{\bar{Y}_{4}^{m_{i}}}{\prod_{h=1}^{8} \bar{Y}_{4}^{m_{h}}} \prod_{j=1, j \neq i}^{8}\left(\frac{1}{\bar{Y}_{j}}-\frac{1}{\bar{Y}_{i}}\right)^{-m_{j}} F_{Y l}\left(Y_{0}\right) .
\end{gathered}
$$

3.3. Case III. In Figure 6, $Y_{1}$ are $\mathrm{SNR}$ of receiving signal at R1, $Y_{2}, Y_{3}$ are SNR of receiving signal at R2, $Y_{4}, Y_{5}$ are receiving signal SNR at R3, and $Y_{6}, Y_{7}$ and $Y_{8}$ are receiving signal SNR at $\mathrm{D}$.

(1) Identical- $m$ :

$$
\begin{aligned}
& F_{\text {out III }}=\left(\frac{m z}{\bar{Y}_{1}}\right)^{3 m} \frac{\Gamma(m)}{\Gamma(3 m)} \\
& \times\left[\frac{(3 m-1) !}{\left(m / \bar{z}_{3}\right)^{3 m}}-\exp \left(-\left(\frac{m}{\bar{Y}_{1}}\right) Y_{0}^{k}\right)\right. \\
& \left.\times \sum_{k=0}^{3 m-1} \frac{(3 m-1) !}{k !} \frac{Y_{0}^{k}}{\left(m / \bar{Y}_{1}\right)^{4 m-k}}\right] \\
& +\left(\frac{m z}{\bar{Y}_{1}}\right)^{7 m} \frac{\Gamma(m)}{\Gamma(3 m)} \\
& \times\left[\frac{(7 m-1) !}{\left(m / \bar{z}_{3}\right)^{7 m}}-\exp \left(-\left(\frac{m}{\bar{Y}_{2}}\right) Y_{0}^{k}\right)\right. \\
& \left.\times \sum_{k=0}^{7 m-1} \frac{(7 m-1) !}{k !} \frac{Y_{0}^{k}}{\left(m / \bar{Y}_{2}\right)^{8 m-k}}\right] \\
& *\left[1-\left(\frac{m z}{\bar{Y}_{1}}\right)^{3 m} \frac{\Gamma(m)}{\Gamma(3 m)}\right. \\
& \times\left[\frac{(3 m-1) !}{\left(m / \bar{z}_{3}\right)^{3 m}}-\exp \left(-\left(\frac{m}{\bar{Y}_{1}}\right) Y_{0}^{k}\right)\right. \\
& \left.\left.\times \sum_{k=0}^{3 m-1} \frac{(3 m-1) !}{k !} \frac{Y_{0}^{k}}{\left(m / \bar{Y}_{1}\right)^{4 m-k}}\right]\right] \\
& +\left[1-\left(\frac{m z}{\bar{Y}_{1}}\right)^{3 m} \frac{\Gamma(m)}{\Gamma(3 m)}\right. \\
& \times\left[\frac{(3 m-1) !}{\left(m / \bar{z}_{3}\right)^{3 m}}-\exp \left(-\left(\frac{m}{\bar{Y}_{1}}\right) Y_{0}^{k}\right)\right. \\
& \left.\left.\times \sum_{k=0}^{3 m-1} \frac{(3 m-1) !}{k !} \frac{Y_{0}^{k}}{\left(m / \bar{Y}_{1}\right)^{4 m-k}}\right]\right]
\end{aligned}
$$




$$
\begin{aligned}
& *\left[1-\left(\frac{m z}{\bar{Y}_{1}}\right)^{7 m} \frac{\Gamma(m)}{\Gamma(3 m)}\right. \\
& \times\left[\frac{(7 m-1) !}{\left(m / \bar{z}_{3}\right)^{7 m}}-\exp \left(-\left(\frac{m}{\bar{Y}_{2}}\right) Y_{0}^{k}\right)\right. \\
& \left.\left.\times \sum_{k=0}^{7 m-1} \frac{(7 m-1) !}{k !} \frac{Y_{0}^{k}}{\left(m / \bar{Y}_{2}\right)^{8 m-k}}\right]\right] \\
& *\left(\frac{m z}{\bar{Y}_{1}}\right)^{7 m} \frac{\Gamma(m)}{\Gamma(3 m)} \\
& \times\left[\frac{(7 m-1) !}{\left(m / \bar{z}_{3}\right)^{7 m}}-\exp \left(-\left(\frac{m}{\overline{\bar{Y}}_{2}}\right) Y_{0}^{k}\right)\right. \\
& \left.\times \sum_{k=0}^{7 m-1} \frac{(7 m-1) !}{k !} \frac{Y_{0}^{k}}{\left(m / \bar{Y}_{2}\right)^{8 m-k}}\right] \\
& +\left[1-\left(\frac{m z}{\bar{Y}_{1}}\right)^{3 m} \frac{\Gamma(m)}{\Gamma(3 m)}\right. \\
& \times\left[\sum \frac{(3 m-1) !}{\left(m / \bar{Y}_{1}\right)^{3 m}}-\exp \left(-\left(\frac{m}{\overline{\bar{Y}}_{1}}\right) Y_{0}^{k}\right)\right. \\
& \left.\left.\times \sum_{k=0}^{3 m-1} \frac{(3 m-1) !}{k !} \frac{Y_{0}^{k}}{\left(m / \bar{Y}_{1}\right)^{4 m-k}}\right]\right] \\
& *\left[1-\left(\frac{m z}{\bar{Y}_{2}}\right)^{7 m} \frac{\Gamma(m)}{\Gamma(3 m)}\right. \\
& \times\left[\frac{(7 m-1) !}{\left(m / \bar{Y}_{2}\right)^{7 m}}-\exp \left(-\left(\frac{m}{\bar{Y}_{2}}\right) Y_{0}^{k}\right)\right. \\
& \left.\left.\times \sum_{k=0}^{7 m-1} \frac{(7 m-1) !}{k !} \frac{Y_{0}^{k}}{\left(m / \bar{Y}_{2}\right)^{8 m-k}}\right]\right] \\
& *\left[1-\left(\frac{m z}{\bar{Y}_{4}}\right)^{7 m} \frac{\Gamma(m)}{\Gamma(3 m)}\right. \\
& \times\left[\frac{(7 m-1) !}{\left(m / \bar{Y}_{4}\right)^{7 m}}-\exp \left(-\left(\frac{m}{\bar{Y}_{4}}\right) Y_{0}^{k}\right)\right. \\
& \left.\left.\times \sum_{k=0}^{7 m-1} \frac{(7 m-1) !}{k !} \frac{Y_{0}^{k}}{\left(m / \bar{Y}_{4}\right)^{8 m-k}}\right]\right] \\
& \text { * }\left[1-\left(\frac{m z}{\bar{Y}_{4}}\right)^{11 m} \frac{\Gamma(m)}{\Gamma(11 m)}\right.
\end{aligned}
$$

(2) Nonidentical $m$ :

$F_{\text {out III }}$

$$
\begin{aligned}
& =\sum_{i=1}^{4} \sum_{k=1}^{m_{i}} \frac{\bar{Y}_{1}^{m_{i}}}{\prod_{h=1}^{8} \bar{Y}_{1}^{m_{h}}} \prod_{j=1, j \neq i}^{4}\left(\frac{1}{\bar{Y}_{j}}-\frac{1}{\bar{Y}_{i}}\right)^{-m_{j}} F_{Y l}\left(Y_{0}\right) \\
& +\left[1-\sum_{i=1}^{4} \sum_{k=1}^{m_{i}} \frac{\bar{Y}_{1}^{m_{i}}}{\prod_{h=1}^{8} \bar{Y}_{1}^{m_{h}}} \prod_{j=1, j \neq i}^{4}\left(\frac{1}{\overline{\bar{Y}}_{j}}-\frac{1}{\bar{Y}_{i}}\right)^{-m_{j}} F_{Y l}\left(Y_{0}\right)\right] \\
& * \sum_{i=1}^{8} \sum_{k=1}^{m_{i}} \frac{\bar{Y}_{2}^{m_{i}}}{\prod_{h=1}^{8} \bar{Y}_{2}^{m_{h}}} \prod_{j=1, j \neq i}^{8}\left(\frac{1}{\bar{Y}_{j}}-\frac{1}{\bar{Y}_{i}}\right)^{-m_{j}} F_{Y l}\left(Y_{0}\right) \\
& +\left[1-\sum_{i=1}^{4} \sum_{k=1}^{m_{i}} \frac{\bar{Y}_{1}^{m_{i}}}{\prod_{h=1}^{8} \bar{Y}_{1}^{m_{h}}} \prod_{j=1, j \neq i}^{4}\left(\frac{1}{\bar{Y}_{j}}-\frac{1}{\bar{Y}_{i}}\right)^{-m_{j}} F_{Y l}\left(Y_{0}\right)\right] \\
& \text { * }\left[1-\sum_{i=1}^{8} \sum_{k=1}^{m_{i}} \frac{\bar{Y}_{2}^{m_{i}}}{\prod_{h=1}^{M} \bar{Y}_{2}^{m_{h}}} \prod_{j=1, j \neq i}^{8}\left(\frac{1}{\bar{Y}_{j}}-\frac{1}{\bar{Y}_{i}}\right)^{-m_{j}} F_{Y l}\left(Y_{0}\right)\right] \\
& * \sum_{i=1}^{8} \sum_{k=1}^{m_{i}} \frac{\bar{Y}_{4}^{m_{i}}}{\prod_{h=1}^{8} \bar{Y}_{4}^{m_{h}}} \prod_{j=1, j \neq i}^{8}\left(\frac{1}{\bar{Y}_{j}}-\frac{1}{\bar{Y}_{i}}\right)^{-m_{j}} F_{Y l}\left(Y_{0}\right) \\
& +\left[1-\sum_{i=1}^{4} \sum_{k=1}^{m_{i}} \frac{\bar{Y}_{1}^{m_{i}}}{\prod_{h=1}^{8} \bar{Y}_{1}^{m_{h}}} \prod_{j=1, j \neq i}^{4}\left(\frac{1}{\bar{Y}_{j}}-\frac{1}{\bar{Y}_{i}}\right)^{-m_{j}} F_{Y l}\left(Y_{0}\right)\right] \\
& \text { * }\left[1-\sum_{i=1}^{8} \sum_{k=1}^{m_{i}} \frac{\bar{Y}_{2}^{m_{i}}}{\prod_{h=1}^{M} \bar{Y}_{2}^{m_{h}}} \prod_{j=1, j \neq i}^{8}\left(\frac{1}{\overline{\bar{Y}}_{j}}-\frac{1}{\bar{Y}_{i}}\right)^{-m_{j}} F_{Y l}\left(Y_{0}\right)\right] \\
& \text { * }\left[1-\sum_{i=1}^{8} \sum_{k=1}^{m_{i}} \frac{\bar{Y}_{4}^{m_{i}}}{\prod_{h=1}^{8} \bar{Y}_{4}^{m_{h}}} \prod_{j=1, j \neq i}^{8}\left(\frac{1}{\overline{\bar{Y}}_{j}}-\frac{1}{\overline{\bar{Y}}_{i}}\right)^{-m_{j}} F_{Y l}\left(Y_{0}\right)\right] \\
& * 1-\sum_{i=1}^{12} \sum_{k=1}^{m_{i}} \frac{\bar{Y}_{6}^{m_{i}}}{\prod_{h=1}^{12} \bar{Y}_{6}^{m_{h}}} * \prod_{j=1, j \neq i}^{12}\left(\frac{1}{\bar{Y}_{j}}-\frac{1}{\bar{Y}_{i}}\right)^{-m_{j}} F_{Y l}\left(Y_{0}\right) \text {. }
\end{aligned}
$$

\section{Numerical Analysis}

In this section, we show numerical results of the analytical outage probability of train-to-train communication model in three cases. We plot the performance curves in terms of average signal-to-noise ratio (SNR) and also show computer simulation results for verification. 




FIGURE 7: Outage probability of train-to-train communication in case I when $m=1$ or $m=2$.

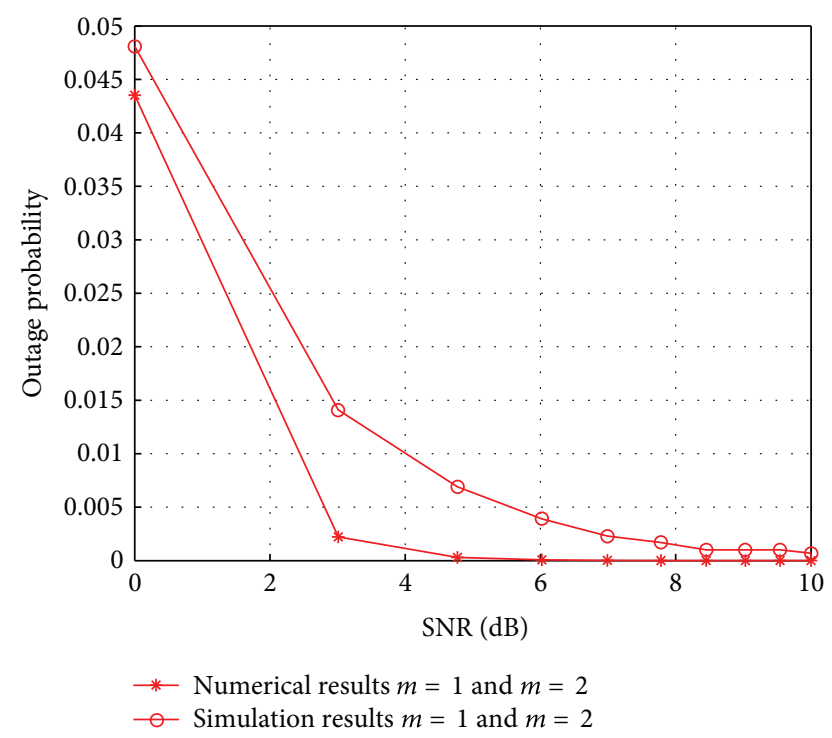

FIGURE 8: Outage probability of train-to-train communication in case I when $m=3$.

According to the previous research, when the receiving SNR is $10 \mathrm{~dB}$, the Bit Error rate (BER) is $10^{-6}$ which satisfies the communication requirements of high-speed railway set by UIC. Therefore, outage performances at SNR $=10 \mathrm{~dB}$ are crucial to the research of train-to-train communication model. If the wireless communication link in railway is disrupted, the railway safety is threatened severely. Figures 7,9 , and 11 show the numerical and simulation results of outage probability of train-to-train communication model versus SNR in case I, case II, and case III when $m=1$ or $m=2$. For $m=1$ in Nakagami- $m$ channel, the channel approximates Rayleigh channel, while for $m=2$ the channel is Rice channel [14]. Because the condition of propagation channel is increasingly becoming better with the increment of parameter $m$, the outage performances of Rayleigh channel are not superior to those of Rice channel. As SNR is $10 \mathrm{~dB}$, in Rayleigh channel, the outage probabilities of case I, case II,



FIGURE 9: Outage probability of train-to-train communication in case II when $m=1$ or $m=2$.

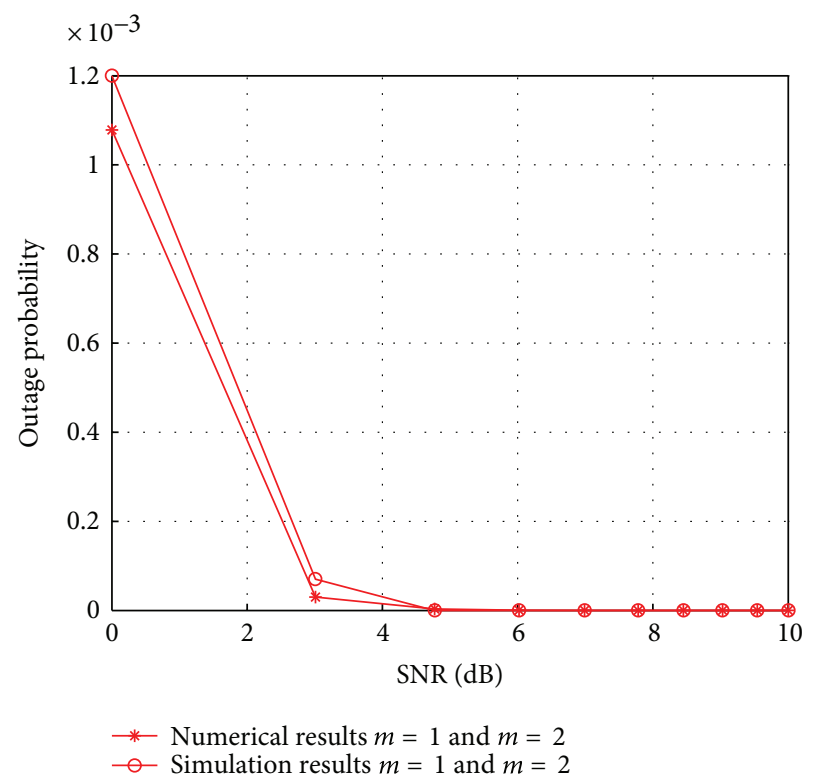

FIGURE 10: Outage probability of train-to-train communication in case II when $m=3$.

and case III are, $0.02,0$, and 0 , respectively, while those of case I, case II, and case III are all 0 in Rice channel. AS for case I in Rayleigh channel, the outage probability is 0.02 , which is bad for wireless communication. But the $m$ parameter in highspeed railway is usually bigger than $m=2$ in fact [14]. Figures 8,10 , and 12 show the numerical and simulation results of outage probability of train-to-train communication model versus SNR in case I, case II, and case III when $m=1$ and $m=2$. The outage probability is $7 * 10^{-4}, 0$, and 0 for three cases at $\mathrm{SNR}=10 \mathrm{~dB}$. Low outage probability contributes to the normal receipt of transmit signal and is rewarding to detection and decision of signal. 


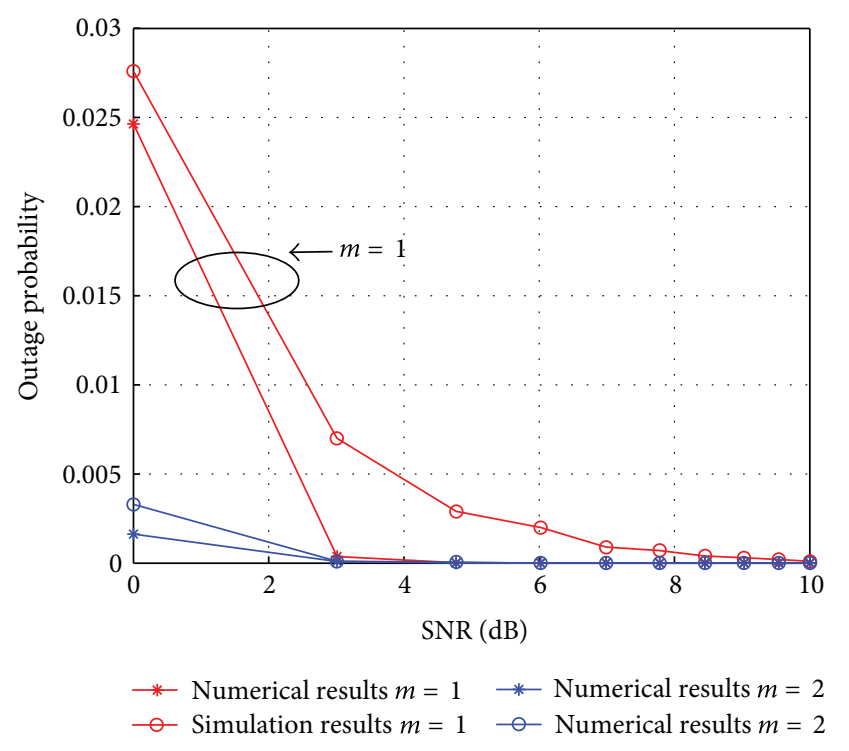

FIGURE 11: Outage probability of train-to-train communication in case III when $m=1$ or $m=2$.

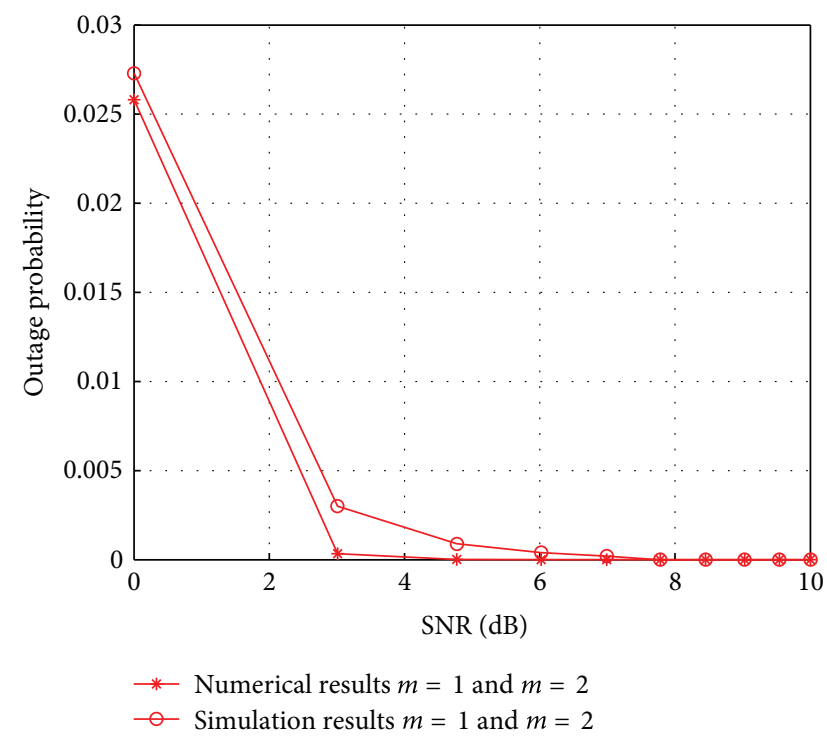

FIGURE 12: Outage probability of train-to-train communication in case III when $m=1$ and $m=2$.

\section{Conclusion}

This paper analyzes the end-to-end outage performance of high-speed-railway train-to-train communication model over independent identical and nonidentical Nakagami- $m$ channels. The mechanism of such communication among trains can be divided into three cases based on occurrence of possible-occurrence relay trains. Numerical and simulation analysis shows that the outage probability of train-to-train communication model in three cases is approximately 0 over Nakagami- $m(m \geqslant 2)$ channel in high-speed railway, which ensures the normal receipt of transmit signal.

\section{Acknowledgments}

This research was supported by the National Natural Science Foundation of China under Grant no. 61222105 the Program for New Century Excellent Talents in University under Grant NCET-09-0206, Fundamental Research Funds for the Central Universities under Grant nos. 2010JBZ008, 2012YJS017, 2012YJS001, and no. 2011YJS010, Key Project of State Key Lab under Grant no. RCS2011ZZ008, National Natural Science Foundation of China under Grant 60830001, Beijing Natural Science Foundation under Grant 4112048, and Program for Changiiang Scholars and Innovative Research Team in University under Grant no. IRT0949.

\section{References}

[1] J. M. Zhang and B. N. Han, "Analysis and calculation of the scheduled waiting time for the train route," in Proceedings of the 4th International Conference on Intelligent Computation Technology and Automation (ICICTA '11), vol. 1, pp. 54-58, March 2011.

[2] T. Strang, M. M. Zu Hörste, and X. G. Gu, "A railway collision avoidance system exploiting ad-hoc inter-vehicle communication and GALILEO," in Proceedings of the 13th World Congress and Exhibition on Intelligent Transportation Systems and Services, pp. 51-56, London, UK, October 2006.

[3] C. R. García, A. Lehner, T. Strang, and M. Röckl, "Comparison of collision avoidance systems and applicability to rail transport," in Proceedings of the 7th International Conference on Intelligent Transport Systems Telecommunications (ITST '07), pp. 1-6, 2007.

[4] C. R. García, A. Lehner, T. Strang, and K. Frank, "Channel model for train to train communication using the $400 \mathrm{MHz}$ band," in Proceedings of the IEEE Vehicular Technology Conference, pp. 3082-3086, May 2008.

[5] C. R. García, A. Lehner, and T. Strang, "A broadcast vehicleto-vehicle communication system in railway environments," in Proceedings of the 1st Annual International Symposium on Vehicular Computing Systems, pp. 70-76, 2008.

[6] A. Lehner, C. Rico-García, E. Wige, and T. Strang, "A multibroadcast communication system for high dynamic vehicular ad-hoc networks," in Proceedings of the International Conference on Ultra Modern Telecommunications and Workshops (ICUMT '09), pp. 1-6, October 2009.

[7] C. R. García, A. Lehner, T. Strang, O. Heirich et al., "Measurement and analysis of the direct train-to-train propagation channel in the $70 \mathrm{~cm}$ UHF-band," in Proceedings of the $3 \mathrm{rd}$ International Workshop on Communication Technologies for Vehicles, vol. 6596, pp. 45-57.

[8] P. Zhao, The Operation and Reorganization of the High Speed Train, China Railway, 2009.

[9] X. Cheng, C. X. Wang, D. I. Laurenson, S. Salous, and A. V. Vasilakos, "An adaptive geometry-based stochastic model for non-isotropic MIMO mobile-to-mobile channels," IEEE Transactions on Wireless Communications, vol. 8, no. 9, pp. 4824-4835, 2009.

[10] X. Cheng, C.-X. Wang, H. Wang, X. Gao et al., "Cooperative MIMO channel modeling and multi-link spatial correlation properties," IEEE Journal on Selected Areas in Communications, vol. 30, no. 2, pp. 388-396, 2012. 
[11] Z. D. Zhong, B. Ai, and Q.Y. Liu, Applications of Fundamental Theory of Railway Digital Mobile Communication System (GSM), Tsing Hua University Press, 2009.

[12] S. Gradshteyn and I. M. Ryzhik, Table of Integrals, Series, and Products, Academic Press, New York, NY, USA, 6th edition, 2000.

[13] G. K. Karagiannidis, N. C. Sagias, and T. A. Tsiftsis, "Closedform statistics for the sum of squared Nakagami-m variates and its applications," IEEE Transactions on Communications, vol. 54, no. 8, pp. 1353-1359, 2006.

[14] R. S. He, Z. D. Zhong, B. Ai, and J. W. Ding, "An empirical path loss model and fading analysis for high-speed railway viaduct scenarios," IEEE Antennas Wireless Propagation Letters, vol. 10, pp. $808-812$. 

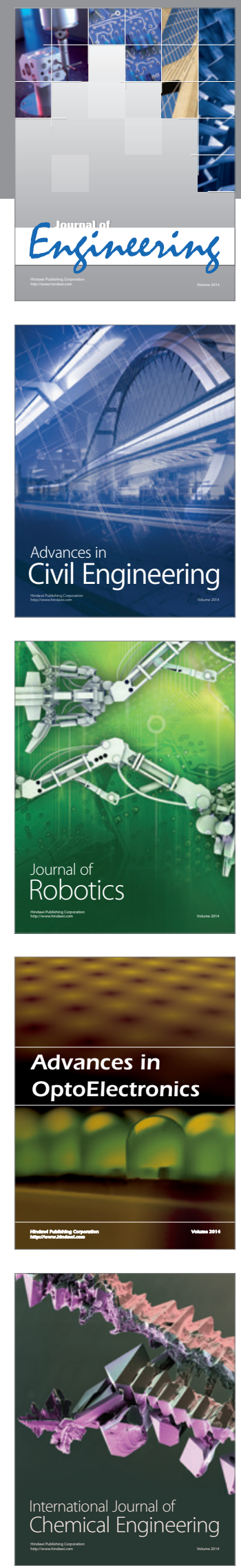

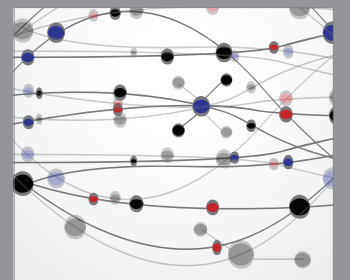

The Scientific World Journal
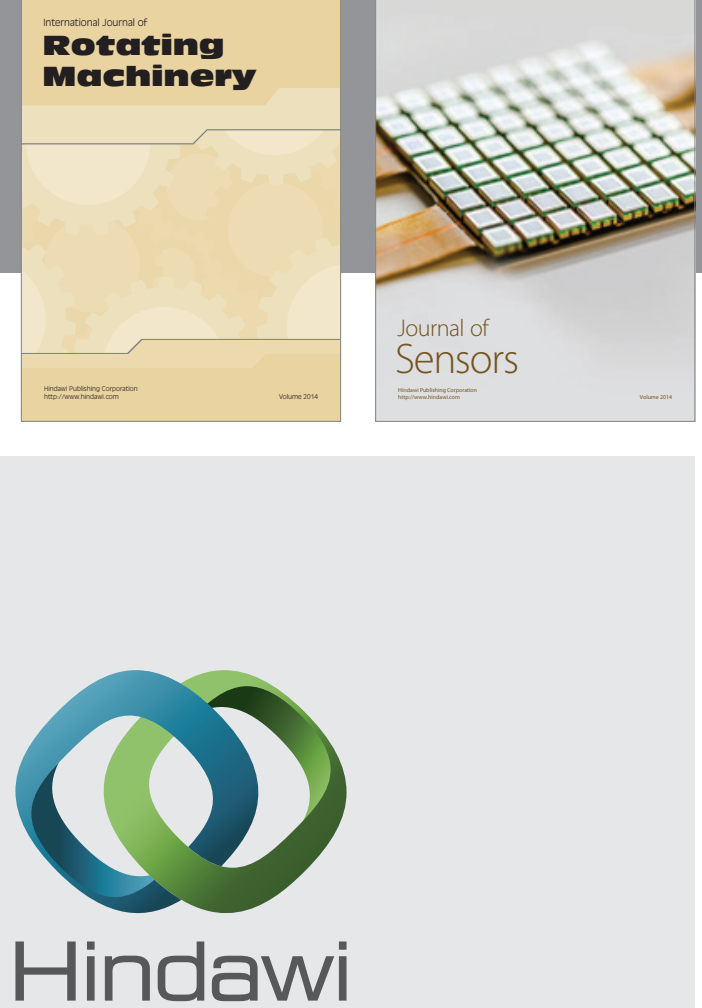

Submit your manuscripts at http://www.hindawi.com

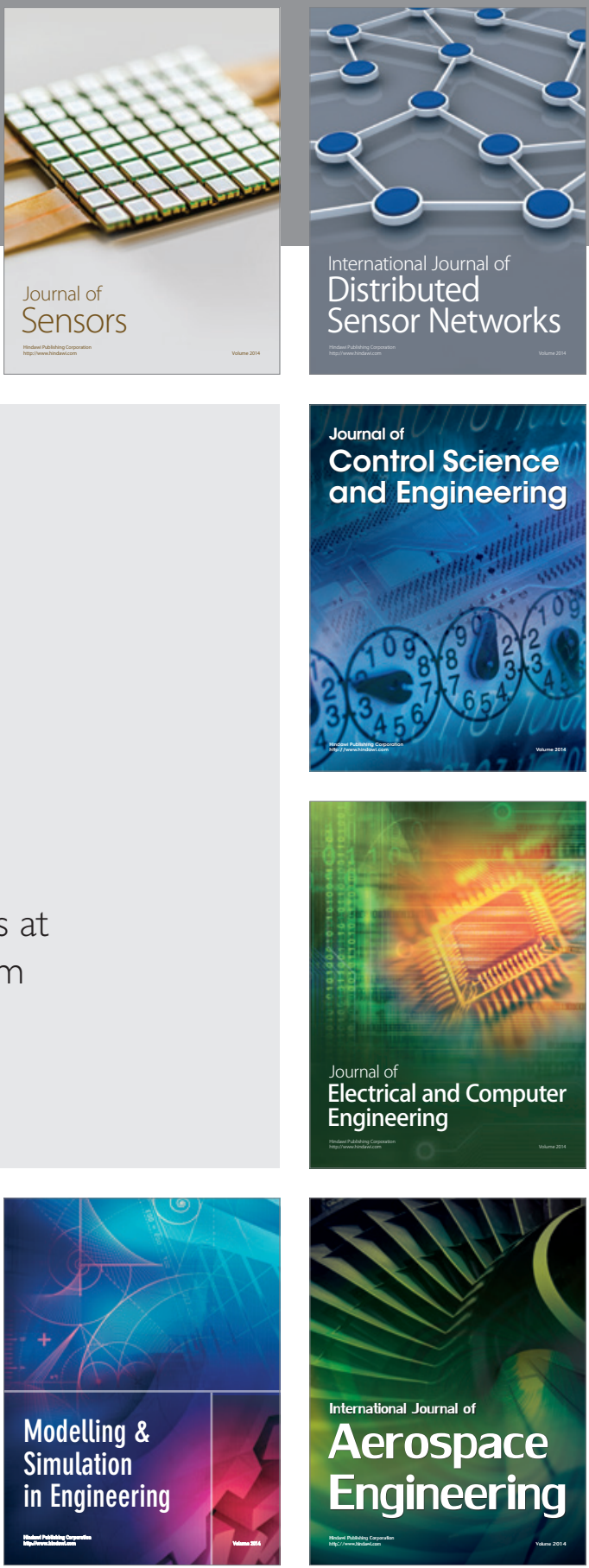

Journal of

Control Science

and Engineering
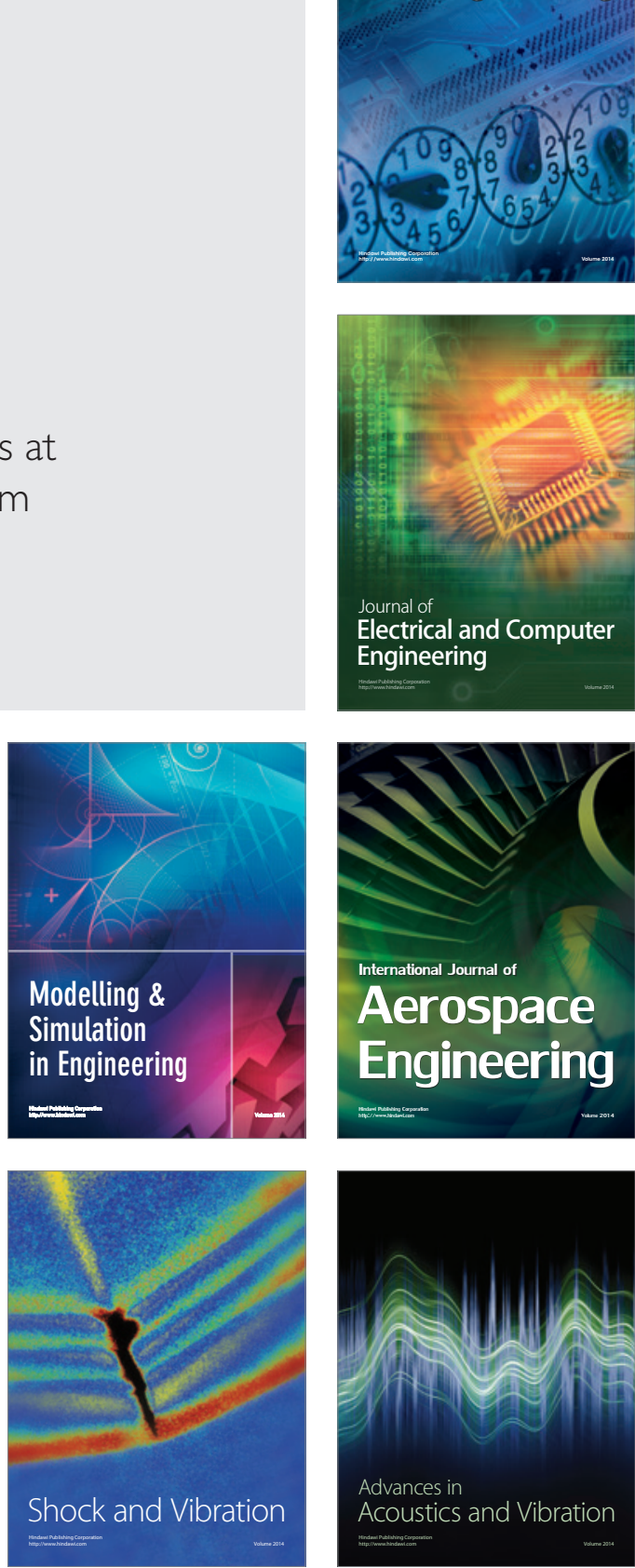